Atıf Bilgisi: Çetin, N. S. (2021). Afroamerikanische burgerrechtsbewegungen in USA zwischen 1956 -1968 und ein burgerrechtsaktivist Martin Luther King JR.: Die artikelinhaltsanalyse von der Arbeiter zeitung. $\dot{I N I F}$ E- Dergi, 6(1), 402-425.

\title{
AFROAMERIKANISCHE BURGERRECHTSBEWEGUNGEN IN USA ZWISCHEN 1956 -1968 UND EIN BURGERRECHTSAKTIVIST MARTIN LUTHER KING JR.: DIE ARTIKELINHALTSANALYSE VON DER ARBEITER ZEITUNG
}

\author{
Öğr. Gör. Dr. Nida Sümeyya ÇETiN* \\ DOI: 10.47107/inifedergi.888634
}

\author{
Araștırma Makalesi $^{* *}$ \\ Başvuru Tarihi: 01.03.2021 \\ Kabul Tarihi: 13.04.2021
}

\section{Zusammenfassung}

Die Bürgerrechtsbewegungen in den USA waren in den sechziger Jahren ein weltweit bekanntes Thema. Die Aktionen durch die gewaltlose Methode wie Gandhis wurde von verschiedenen politischen Segmenten und von weltweiten Medien mit Erstaunen und Zufriedenheit begrüßt. In Medien und vor allem in den Zeitungen wurden sowohl über die offiziellen Seiten der Aktionen als auch über die Ursachen und den Verlauf der Bewegungen berichtet. Somit stehen hier die Berichterstattungen in Bezug auf die ganzen Themenprozesse und auch die Art und Weise der Präsentation im Mittelpunkt. Durch die Berichterstattungen wird eine ausgeprägte Vorschau über die Ereignisse ermöglicht. Hinsichtlich der Interpretationen der Bürgerrechtsbewegungen und des Verstehprozesses der 68er Bewegungen spielen die Medien, für die vorliegende Arbeit vor allem die Zeitung eine große Rolle. İn der Artikel wird die Berichterstattung der sozial-demokratische Arbeiter Zeitung untersucht und dann ein Interpretationsversuch gestartet. Der erste Schwerpunkt liegt auf dem Teil „Forschungsstellung“. In erste Linie wird kurz versucht, die Vernunft des amerikanischen Herrschaftssystems zu verstehen. Danach wird die kurze Geschichte der Afroamerikaner im Zusammenhang mit den Bewegungen erläutert. Als letztes werden die Bürgerrechtsbewegungen durch die bedeutende Persönlichkeit Martin Luther Kings und seine gewaltlosen Methoden in der chronologischen Reihenfolge dargestellt. Der zweite Schwerpunkt liegt auf dem Theorieteil. In diesem Teil wird die Nachrichtenwerttheorie mit ihren Ansätzen und Definitionen gefördert. Abschließend werden die Nachrichtenfaktoren mit ihren Definitionen bzw. Dimensionen nach heutigen medialen Aspekten dargelegt. Der dritte Schwerpunkt der Arbeit erfolgt in Form einer empirischen Analyse. Im Rahmen einer quantitativen Inhaltsanalyse soll überprüft werden, in welcher Form die Arbeiter Zeitung in den Berichterstattungen über die Bürgerrechtsbewegungen vermittelt hat.

Schlüsselwörter: Medien, Bürgerrechtsbewegung, Martin Luther King, Nachrichtenwerte

\section{ABD'DE 1956-1968 ARASINDAKİ AFROAMERİKAN VATANDASSLIK HAREKETLERI VE BİR VATANDAŞLIK HAKLARI AKTIVISTI MARTIN LUTHER KING JR.: İ̧̧Çi GAZETESINIIN HABER İÇERIK ANALIZİ}

\section{$\ddot{O} \mathbf{z}$}

Amerika Birleşik Devletleri'ndeki vatandaşlık hakları hareketleri, 1960'larda tüm dünya tarafindan bilinen bir konuydu. Gandhi gibi şiddetsiz yöntem içeren eylemler, çeşitli siyasi kesimler ve dünya medyasından şaşkınlık ve memnuniyetle karşılanmıştır. Medya ve özellikle gazetelerde hem sivil itaatsizlik hareketlerinin seyri ve sebepleri hem de eylemlerin resmi açıklamaları hakkında da sürekli haberler yapılmıştır. Yapılan bu haberlerin odak noktasında eylem süreçlerinin tamamı ve sunum biçimleri yer almıştır. O dönemde haberler aracılığı ile olaylara yönelik net bir şekilde önizleme sağlanmıştır. Vatandaşlık hakları hareketlerinin yorumlanmasında ve 68 hareketlerini anlama sürecinde medya, çalışma açısından ise özellikle gazeteler büyük bir rol oynamıştır. Bu çerçevede çalışmada, sosyal demokrat işçi gazetesinin haber yapısı incelenmiş ve ardından yorumlanmıştır. Çalışmanın ilk odak noktası, araştırma kısmının yapıldığı teorik kısmıdır. Teori kısmında ilk olarak Amerikan yönetim sisteminin siyahi vatandaşlarına yönelik

\footnotetext{
* İnönü Üniversitesi, İletişim Fakültesi, Gazetecilik Bölümü, E-mail: nida.cetin @inonu.edu.tr, ORCID-ID: 0000-00019752-7797

** Yazar / yazarlar, makalede araştırma ve yayın etiğine uyulduğuna ve kullanılan fikir ve sanat eserleri için telif hakları düzenlemelerine riayet edildiğine yönelik beyanda bulunmuştur.
} 
duruşunu anlamak için kısa bir girişimde bulunulmuştur. Ardından Afrika kökenli Amerikalıların düzenledikleri vatandaşlık hakları hareketleri ilgili kısa tarihçe anlatılmıştır. Son olarak, sivil haklar hareketleri, Martin Luther King'in seçkin figürü ve şiddetsiz yöntemleri, kronolojik sırayla sunulmuştur. Yöntem kısmında ise; ilk olarak haber değer teorisi yaklaşımlar ve tanımlar çerçevesinde açıklanmıştır. Bu bölümde haber faktörleri; tanımları ve boyutları ile günümüz medya anlayışı çerçevesinde ortaya konulmaya çalışılmıştır. Ampirik bölümde nitel içerik analizi çerçevesinde seçilen gazetenin eylemleri içeren haber yapısının nasıl olduğu ve hangi biçimde aktarıldığı anlaşılmaya çalışılmıştır.

Anahtar Kelimeler: Medya, Vatandaşlık Hareketi, Martin Luther King, Haber Değerleri

\section{Einleitung}

Es gab früher Rechte, die sehr tatkräftig waren, wenn sich etwas ereignete. Zudem wurden diese Rechte als sehr wertvoll und wichtig geschätzt und die Menschen versuchten sich daran zu halten. Kriege, Proteste, Widerstände waren die Gründe dafür, dass solche Rechte entstanden und auch in die Tat umgesetzt wurden. Es war sogar so, dass viele Menschen, um diese Rechte aufrecht zu erhalten ohne Zögern ihr Leben gegeben hätten. Mit der Zeit nahmen dann diese Arten von Unheil und Konflikte in der Geschichtsszene ihren Platz ein und die dadurch entstandenen Rechte wurden dann unter dem Sammelbegriff „Menschenrechte“ zusammengefasst. Sicher ist jedoch, dass die Menschenrechte immer noch ein vieldiskutiertes Thema sind und leider auch von allen Seiten vernachlässigt werden.

In 20. Jahrhundert ging es auch um die Rechtsbewegungen und man dürfte ja sagen, dass die noch ein Jahrhundert mit diesen Erschütterungen begonnen und leider noch so beendet hat. Am Anfang des Jahrhunderts setzte in Literaturen noch ein Begriff ein, "Ziviler Ungehorsam". Rechtsbewegungen haben mit den Gandhis Widerstand, also sogenannte "Ziviler Ungehorsam" eine neue Perspektive gewonnen und waren für jede Art von Widerstand ein richtiges und wirkungsvolles Beispiel. Nach Gandhis „Gewaltloser Widerstand"-Taktik verhielt sich auch die schwarze Bevölkerung in den USA gegen die US-amerikanische Regierung. eine weitere Reaktion bezüglich Widerstands von Seiten der schwarzen Menschen in den USA entwickelt. zweite enorme Reaktion sich auf der Seite Schwarzen Menschen in USA durch Gandhis Technik gegen die US- amerikanische Regierung stattgefunden. Ab 1955 haben die Bürgerrechtsbewegungen, die in den USA stattgefunden haben, sowohl in der US-Geschichte als auch auf der ganzen restlichen Welt eine große Bahn geschaffen. Auf ganzer Welt wurde die Rassentrennung als ein großes gesellschaftliches Problem betrachtet.

Das waren ein Symbol und eine Hoffnung für die unterdrückten Minderheiten auf der ganzen Welt. Auffallend ist jedoch, dass diese Bewegungen im Rahmen des „Zivilen Ungehorsam“ erfolgreich waren, obwohl ihre Gegner stark und gewalttätig waren. Diese Gegner kannten niemals Hemmungen, während sie die schwarzen Menschen in ihrem eigenen Land getötet und diese Menschen wie Sklaven oder Leibeigene behandelt hatten.

Heutzutage ist das Rassentrennungsproblem noch immer die politische Debatte in vielen Ländern. Die Schwarzen Menschen und ihre sozialen Situationen in der Gesellschaft sind noch nicht klar festgelegt. Wie man schon gesagt hat, „die Schwarzen Menschen haben ein schwarzes Glück, wie ihre schwarze Hautfarbe!“

\section{Afrika in Amerika! Geschichte der Schwarzen in den USA}

Um die Stellung der Schwarzen in der amerikanischen Geschichte besser verständlich zu machen, muss zuerst auf das amerikanische System und sein Rechtsverständnis von seinen Anfängen bis zur Gegenwart eingegangen werden. Aus diesem Grund wird hier zunächst das amerikanische Herrschaftssystem überblicksartig dargestellt. 


\section{1. Überblick über das Amerikanische Herrschaftssystem}

Wenn man durchschaut hat, unter welchen Bedingungen die Vereinigten Staaten von Amerika begründet wurden, versteht man auch die Gründe ihrer imperialistischen Verbreitungspolitik besser. Um neue ökonomische Ressourcen und Humankapital zur Befriedigung der eigenen Bedürfnisse zu erschließen und um in der Weltpolitik sowohl ideologisch als auch kulturell Einfluss zu gewinnen, versuchten sie sich durch immer neue Methoden in verschiedenen Ländern und Kontinenten zu etablieren. Das Hauptziel dieses Systems war und ist es "die Welt zu einem willfährigen, vor allem aber freiwilligem Abnehmer amerikanischer Produkte zu machen." (Weber, 1996, s.2). Die wichtigsten gesellschaftlichen Prinzipien, die von den Vereinigten Staaten zu Propagandazwecken verwendet wurden, wie Demokratie, Freiheit, Gleichheit, etc., haben sich in den Vereinigten Staaten selbst erst spät durchgesetzt. Nach der Ausrufung der ersten Republik der Welt durch die Unabhängigkeitserklärung am 4. Juli 1776 wurde die amerikanische Verfassung und wurde die Gewaltenteilung in der Verfassung verankert.

1791 wurden in den Vereinigten Staaten allgemeine Menschenrechte wie Religionsfreiheit, Presse- und Redefreiheit mit der Bill of Rights anerkannt. Doch die Bundesstaaten wurden in staatliche Gerichtsverfahren nicht eingeschlossen. Das heißt, „die police power blieb weiterhin bei den Einzelstaaten, was teilweise vor allem im Süden der USA zu harten Diskriminierungen gegenüber bestimmten Bevölkerungsteilen führte.“ (Weber, 1996, s. 25). Die Diskriminierung traf besonders die schwarze Bevölkerung. Das Wahlsystem der USA ist ein bis heute heftig diskutiertes Thema. Im Zweiparteiensystem der USA gibt es ein spezielles Registriersystem für Wähler, wodurch den Wählern selbst die Verantwortung für die Möglichkeit zu wählen übertragen wird. Das Problem manifestiert sich in den Wahlen des Präsidenten und des Kongresshauses geringe Wahlbeteiligung und die Machtkämpfe zwischen den Parteien führen zu innenpolitischen Problemen. Soweit es die schwarze Bevölkerung betrifft, wurde im Jahr 1965 mit der Akzeptierung des Voting Rights Act mit der Lösung des Problems begonnen. Mit diesem Gesetz haben die Schwarzen, die bis dahin von den Wahlen ausgeschlossen waren, ihre Wahlrechte erhalten. Man kann sagen, dass das amerikanische System an diesem Problem verzweifelt ist, was seiner Leistungsfähigkeit und seinem Einfluss in der Weltpolitik jedoch keinen Abbruch getan hat.

\subsection{Die Geschichte der Schwarzen in Amerika}

Um die Frage nach den Ursachen der Bürgerrechtsbewegungen in den USA hinreichend beantworten zu können, ist es wichtig, sich die Geschichte der Schwarzen anzusehen. Ihre Geschichte begann mit zwanzig Afrikanern. Sie wurden an die Kolonisten in Jamestown, Virginia verkauft. Bei diesen so genannten ,indentured servants (vertragsgebundene Diener)“ handelte es sich allerdings nicht um Sklaven, sondern um Zwangsarbeiter, die in der Regel nach sieben Jahren wieder freigelassen wurden (Moosbrugger, 2004, s. 1). In der nordamerikanischen Geschichte nehmen die Schwarzen einen festen Platz ein. Für die Weißen stellte ihre Arbeit einen wirtschaftlichen Gewinn dar. Die Sklaven arbeiteten zumeist in der Landwirtschaft, auf den ausgedehnten Reis-, Tabak und Zuckerrohrplantagen (Pressler, 1984, s. 12).

Der Anteil der Gesamtbevölkerung in den Kolonien der Südstaaten wuchs bis ins 19.Jh. nach der ersten Bundesvolkszählung von 1790 lebten in den Südstaaten 697.897 Sklaven, 1810 1.191.354, was eine Erhöhung von mehr als siebzig Prozent bedeutet. Nach dem Unabhängigkeitskrieg bis hinein ins 19. Jahrhundert erlebte die Sklaverei wegen der wachsenden Produktion von/Nachfrage nach Baumwolle in den südlichen Kolonien der Vereinigten Staaten einen massiven Aufschwung und erschien am Anfang des 
19.Jahrhunderts als eine Wirtschaftsnotwendigkeit (Engel, 2006, s. 8). Im 19. Jahrhundert verschlechterte sich die Situation der Schwarzen mit dem wirtschaftlichen Wachstum des Landes. Die verschärften Probleme brachten den gewaltsamen Widerstand mit sich. Die Aufstände in Virginia und Charleston sind nur einige Beispiele davon. Anfangs waren sie auch erfolgreich. Sie wurden von abolitionistischen Vereinigungen geführt, die im Norden aus Protest gegen die im Süden der USA praktizierte Sklaverei entstanden waren (Moosbrugger, 2004, s. 3). Später wurden diese Widerstände gewaltsam beendet.

Die Befreiungsgeschichte hatte mit diesen Aufständen begonnen. Für die Abschaffung der Sklaverei setzten sich auch weiße Amerikaner ein wie W. Loyd Garrison, der erste weiße Publizist, der die Rechte der Schwarzen öffentlich verteidigt hat, oder Henry D. Thoreau, der argumentierte, dass die ungerechten Gesetze oder eine gerechte Sache nur durch zivilen Ungehorsam dem Gewissen der Öffentlichkeit bekannt gemacht werden kann (Suber, 1999). Nach dem Bürgerkrieg von 1860-1865 zwischen den Nordund den Südstaaten mit dem Sieg des Nordens wurde die Sklaverei im ganzen Staatsgebiet aufgehoben. Die Schwarzen waren endlich frei und konnten von ihren Wahlrechten nun auch Gebrauch machen. Aber diese Phase der Befreiung dauerte nicht lange. Im Süden wurde die Rassentrennung nämlich verschärft. Die Rassisten im Süden der USA setzten nach 1865 zur Umgehung der von der föderalen Regierung in Washington verordneten Gleichstellung so genannte „black codes“ ein (Moosbrugger, 2004, s. 3). Mit diesen „codes“ wurde die Etablierung der Rassentrennung und Zwangsarbeit im wirtschaftlichen Bereich erzielt. In der Gründung der Geheimorganisation „Ku-Klux-Klan“ manifestierte sich der unmenschliche Terror gegen die Schwarzen. Der Ku-Klux-Klan wurde zum Symbol der Unterdrückung im Süden.

Am Anfang des 20. Jahrhunderts wurde eine Organisation unter dem Namen, National Association for the Advancement of Colored People' (NAACP- Nationale Vereinigung für die Förderung von farbigen Leuten) gegründet, deren Hauptziele die Beseitigung der Rassenvorurteile, Rechtsgleichheit, Stimmrecht bei den Wahlen, Bildungsund Ausbildungschance waren (Pressler, 1984, s. 20). Die Organisation war legal und ihre Protestformen legalistisch - institutionalisiert angesehen. Die Proteste wurden erst durch die Kirchen, die durch Boykotte und andere Massenaktionen ihre Ziele durchzusetzen bereit waren, ersetzt (Weber, 1996, s. 167). NAACP hatte viele Erfolge, aber ihr größter Erfolg war die Aufhebung der Rassentrennung an Schulen aufgrund von Verfassungswidrigkeit.

Am Beginn des 20.Jahrhunderts verstärkten sich die nationalistischen Strömungen bei den Schwarzen. Sie trauten der so genannten Reformbereitschaft der Weißen nicht. Die Schwarzen verweigerten die amerikanischen Aspekte und wollten ihre afrikanische Identität zurückhaben. Den nationalistischen Schwarzen wie Marcus Garvey, Vorsitzender der Separatisten, geht es um die Bewegung 'zurück nach Afrika'. Bedeutendster Vertreter dieser nationalistischen Strömung in den sechziger Jahren war Malcolm X. des zweiten Krieg veränderte die politischen Dimensionen weltweit. Mit dem Krieg erhofften die ethnischen Minderheiten breitere Freiheiten in den eigenen Ländern zu erringen. Bei den Schwarzen war diese Hoffnung auf die Aufweichung der Rassentrennung gedrückt, weil sie bereits für die amerikanischen Kriegszwecke in den Krieg gezogen waren und gekämpft hatten. Wie Blumberg (1984, s. 34) in ihrem Buch bereits geschrieben hat; "During the war, black Soldiers went abroad again in the name of democracy, experienced segregation in the armed forces and their recreational facilities."

Diese Erwartungen waren nicht ganz in die Brüche gegangen. Die Schwarzen haben einiges an Rechten gewonnen wie z.B. die Aufnahme schwarzer Schüler an den 
Schulen im Süden, wo die strenge Form der Rassentrennung herrscht oder die Erlaubnis frei im Land zu reisen. Aber diese Erfolge der Schwarzen konnten die Rassenunruhen im Süden nicht verhindern. Es kam immer öfter zum Ausbruch von Gewaltakten, wie Bombenanschlägen auf Häuser, Morde und anderes mehr. Nur in die wenigsten fallen wurden die weißen Täter aber angeklagt und kein einziges Mal kam es zu einer Verurteilung wegen Lynchjustiz (Moosbrugger, 2004, s. 30).

\subsection{Die Little Rock Krise}

1957 trat das Bildungsgesetz des Obersten Gerichtes in Little Rock in Kraft, dessen Hauptziel die Integration der schwarzen Studenten an den Schulen zu sichern und bzw. wegzuschauen, was die „offensichtlich Angelegenheiten“" anging (Moosbrugger, 2004, s. 33). Doch der Gouverneur von Arkansas, Orval Faubus, widersetzte sich der USRegierung. Mit dem Inkrafttreten des neuen Gesetzes wurden von der Schulbehörde neun schwarze Studenten für das Studium an der Little Rock Central High School ausgewählt. Der Gouverneur von Arkansas Faubus protestierte dagegen und behauptete, wenn afroamerikanische Studenten versuchen sollten, die Central High School zu betreten, werde es zu blutigen Kämpfen auf der Straße kommen (Engel, 2006, s. 27). Daraufhin griff der Präsident Eisenhower ein und schickte über 1.000 Soldaten nach Arkansas, um die schwarzen Kinder gegen den rassistischen Angriff zu schützen. Mit diesem Fall konnte die US-Regierung die Bürgerrechtsbewegung davon überzeugen, dass sie auf Seite der Schwarzen sei. Das Gesetz gab den Schwarzen zwar nicht ihre Freiheit zurück, aber dennoch wurden die Lösungsversuche der Regierung sowohl im eigenen Land als auch international optimistisch begrüßt.

\section{Bürgerrechtsbewegungen der Schwarzen in den USA}

Im allgemein kann man soziale Bewegungen zum rationalen Problemlösen als objektive soziale Probleme darstellen (Schwarzmeier, 2001, s. 14). In diesem Aspekt wäre es möglich, zu sagen, dass Bürgerrechtsbewegungen der Afroamerikaner zur Problemlösung geschehen waren. Bevor die Zeitgeschichte der Bürgerrechtsbewegungen erwähnt wird, sollte der Begriff "Bürgerrechtsbewegung" kurz dargelegt werden. Bürgerrechtsbewegungen der Afroamerikaner werden die Forderungen im Wertehorizont von Demokratie formuliert und sie stemmten sich bei unzähligen spektakulären Protestaktionen mit gewaltfreiem Widerstand gegen Gewehre. Daneben zogen die Aktivisten die Interaktion zwischen den Strategen der Bewegung und den Reaktionen der Medien in Erwägung und das verstärkte die Protestausbreitung sich überall in Amerika (Butter vd., 2016, s. 18). In diesem Teil wurde das Protestkonzept der Bürgerrechtsbewegungen berührt.

\section{5}

2.1.Wendepunkt für die schwarze Bevölkerung: Busboykott von Montgomery

Um den Montgomery Busboykott genau interpretieren und verstehen zu können, muss man sich in erster Linie die Rolle der Kirchen auf die schwarze Bevölkerung vor Augen führen. Die schwarzen Kirchen förderten die unteren Gesellschaftsschichten finanziell und veranstalteten Programme für Kinder und Rentner. Mit dem Montgomery Boykott übernahmen die Kirchen Organisationen durch die Begabten Führer bedeutende Rolle in der Gesellschaft:

"It demonstrated the ability of black leaders to mobilize all segments of the community-the elite and masses- through their churches, organizations and communication structures and to gain moral and financial support from sympathizers throughout the country." (Blumberg, 1984, s. 41). 
In Montgomery, wo damals 40 Prozent von 120.000 Bewohnern Schwarze waren und in der ein streng rassistisches System in öffentlichen Einrichtungen herrschte, durften Schwarze nur im hinteren Teil der Busse Platz nehmen und mussten selbst diesen den Weißen überlassen, wenn diese keinen anderen Platz im Bus finden konnten (Bahr, 1990, s. 40). Den Wendepunkt in der afroamerikanischen Geschichte führte die Verweigerung einer Schwarzen Frau gegen die Rassendiskriminierung im Bus herbei. Am 1. Dezember 1955 wollte die Sekretärin von NACCP, Rosa Parks, von ihrem Arbeitsplatz nach Hause zurückkehren. Im Bus gab es keine Plätze mehr und der Busfahrer forderte Mrs. Parks auf ihren Platz für einen weißen Passagier freizumachen. Mrs. Parks weigerte sich und blieb sitzen, woraufhin sie inhaftiert wurde und eine Geldstrafe auferlegt bekam. Doch sie weigerte sich auch die Geldstrafe zu bezahlen. Dieses Ereignis gilt allgemein als Anlass bzw. Auslöser für die Bürgerrechtsbewegungen (Moosbrugger, 2004, s. 42).

\section{Boykotts}

2.1.1. „Die Welt stand auf, als Rosa Parks sitzen blieb“1: Die Organisation des

Die Geldstrafe wurde von Edgar Daniel Nixon, dem Präsidenten der NAACP, bezahlt und Rosa Parks wurde freigelassen. Im nächsten Tag wurde die Nachricht in Umlauf gesetzt. Die Pfarrer und die Gewerkschaften trafen sich in der Dexter Avenue Church, in der Kirche M. L. Kings und übertrugen die Organisation des eintägigen BusBoykotts Jo Ann Robinson, der Leiterin des lokalen „Women's Political Council“, die schon ähnliche Solidaritätsaktionen geplant hatte (Moosbrugger, 2004, s. 43). Am 5. Dezember, einem Montag wurde der Boykott mit Erfolg durchgeführt. Tausende Schwarze gingen zu Fuß zu ihren Zielen:

„Black People were walking, thumbing rides, or pilling into cars and taxicabs. None was riding the city buses" (Engel, 2006, s. 27).

Die Aktion war der erste Schritt für die zukünftigen Bürgerrechtsbewegungen. Am gleichen Tag wurde entschied man sich den Boykott unter Beibehaltung der Forderungen $\mathrm{zu}$ verlängern. $\mathrm{Zu}$ diesem Zwecke wurde die MIA- Montgomery Improvement Association- gegründet und der junge Pfarrer Martin Luther King Jr. zu ihrem Präsidenten gewählt. Mit diesem Beschluss begann der Boykott am 8. Dezember in Montgomery. Der Boykott dauerte über ein Jahr lang. Über die Bürgerrechtsbewegungen wurde in nationalen Zeitungen, Zeitschriften und im Fernsehen berichtet. Neben der nationalen Aufmerksamkeit erregte der Boykott auch die internationale Aufmerksamkeit. Die weißen Organisationen setzten Aktionen gegen die Bürgerrechtsbewegungen, attackierten Kirchen und zerbombten Häuser. Martin Luther King und andere Führungsfiguren der Bürgerrechtsbewegung wurden zu Geldstrafen verurteilt. All das war Teil eines politischen Spiels gegen die Rassenintegration in der amerikanischen Gesellschaft. Der politische Gegner versuchte die legitimen Forderungen der schwarzen Bürgerrechtsbewegung nach der Rasseintegration oder auch Desegregation mit aller Gewalt zu verhindern (Weber, 1996, s. 172).

Nach vielen Anklagen und gerichtlichen Prozessen entschied endlich der oberste Gerichtshof am 13. November 1956, dass die von den lokalen und innerstaatlichen Gesetzen Alabamas gutgeheißene Rasentrennung in Bussen gegen die Verfassung verstoße. Damit wurde diese Art der Rassentrennung aufgehoben (Moosbrugger, 2004, s. 47). Am 21. Dezember 1956 endete der 382 Tage dauernde Boykott. Mit ihrem Durchhaltevermögen hatten es die Schwarzen geschafft die Rassendiskriminierung zu besiegen.

\footnotetext{
1 aus einem Lied
} 


\subsubsection{Nach dem Boykott}

Der gewaltfreie Widerstand von Martin Luther King erzeugte weltweites Echo. Seine Überzeugungsfähigkeit und sein Konzept der Gewaltfreiheit machten ihn zum Vorreiter. Nach seinem Vorbild wurden gewaltfreie Rede und gewaltlose Taktik in den Südstaaten eingeführt. Nach dem Boykott rief Martin Luther King die schwarzen Anführer zusammen um mit ihnen die Southern Christian Leadership Conference, kurz SCLC genannt, zu gründen. Als Präsident wurde einstimmig Martin Luther King gewählt. Hauptziel der SCLC war es, die große Masse der Negerbevölkerung für den Freiheitskampf zu interessieren, indem sie das „Montgomery-Modell“ im Süden weiter verbreitete (Oates, 1984, s. 154).

\subsection{Die Sit-in Bewegung 1960}

"We Started Because We Were Tired of Waiting for You to Act." (Sit-In Participant in Chattanooga, TN).

Im Süden existierte die Rassendiskriminierung in Restaurants, Theatern und Kinos weiter. Viele Afroamerikaner erwarteten sich deutliche Fortschritte von Gerichtsverfahren sowie in Montgomery und sie wurden endlich ungeduldig (Moosbrugger, 2004, s. 71). Die afroamerikanischen Studenten vom Nord Carolina „Agricultural and Technical College“, Joseph McNeil, Franklin McCain, David Richmond und Ezell Blair, nahmen sich vor diesen Prozess zu beschleunigen. Mit dieser Absicht gingen Sie am ersten Februar (1960) zum Imbiss Woolworth in Greenboro, wo Schwarzen normalerweise nicht serviert wurde. Nachdem sie etwas gekauft hatten, wollten sie das Mittagsessen einnehmen. Aber es wurde ihnen nicht serviert, weil sie ,Neger' waren. Doch an diesem Tag blieben sie in der Cafeteria sitzen. Bis zum dritten Februar gingen sie täglich in die Cafeteria und blieben dort sitzen. Am dritten Februar waren es bereits 63 Studenten, die sich in dem Imbiss einfanden. Auch weiße Studenten schlossen sich ihnen an. Die Sit-in Bewegung dauerte bis ins Jahr 1961. Während dieser Zeit wurden hunderte Studenten in verschiedenen Städten verhaftet. Viele sit-inners betrieben dennoch weiterhin das gewaltlose Konzept. Ihr Sit-In Konzept beruhte auf Martin Luther Kings Modell der gewaltfreien Aktion. Die SitIn Bewegungen griffen auch auf andere Städte über wie Georgia, Atlanta oder Nashville, wo umfassende Aktionen durchgeführt wurden. Die Aktivisten kämpften nicht nur für Bedienung in Imbisstheken sondern auch für gleiche Behandlung in öffentlichen Gebäuden und Geschäften, für gleiche Wahlrechte und gleiche Chancen auf Bildung.

Die Bewegung endete im September 1961, nachdem in 28 südlichen Städten die Rassendiskriminierung in Imbissen und Restaurants aufgehoben worden war. An der "sitin Bewegung" hatten 70.000 Amerikaner teilgenommen:

„throughout 1960 and the next year about 70,000 participated in thirteen states, and a newspaper in Raleigh noted that the picket line now extends from the dime store to the United States Supreme Court and beyond that to national and world opinion." (Engel, 2006, s. 39).

\subsection{SNCC- Student Nonviolent Coordinating Committee}

"Eine Generation von jungen Leuten ist aus Jahrzehnten des Dunkels ans Licht getreten und steht nackter Staatgewalt gegenüber!"-Martin Luther King (Oates, 1984, s. 89).

Nach dem Erfolg der Sit-in Bewegung hatten die Afroamerikaner, bzw. Martin Luther King und andere Leaders die Idee, ihre Strategie der Gewaltfreiheit durch Studenten im ganzen Land zu verbreiten und so der Segregation mit gewaltloser Aktion 
entgegenzutreten (Oates, 1984, s. 190). Zu diesem Zweck organisierte Martin Luther King eine Studentenkonferenz in Raleigh, North Carolina. Er schlug den Studenten vor unter dem Dach der SCLC eine eigene Organisation zu schaffen. Ella Barker, Zivil- und Bürgerrechtsaktivistin, lehnte diesen Vorschlag jedoch ab und schlug stattdessen vor, eine unabhängige Organisation zu gründen. Die Konferenz traf schließlich die Entscheidung zu einer unabhängigen "Studentenorganisation", die gewaltfreie Aktivitäten in südlichen Städten durchführen sollte.

Am 17. April 1960 begründeten die Studentenleaders die Organisation unter dem Namen „Student Nonviolent Coordinating Committee“ (genannt snick). Später übernahm das SNCC die Führung der alten Organisationen der NACCP und SCLC und sie arbeiteten von da an zusammen (Moosbrugger, 2006, s. 74).

\subsection{Präsidentschaftswahlen 1960}

Die Präsidentschaftswahlen 1960 spielten für die Frage nach der Situation der Millionen Afroamerikaner eine entscheidende Rolle und bestimmten deren Schicksal in den USA. Beide Kandidaten, Richard Nixon und John F. Kennedy, versprachen die Rassenintegration zu sichern. Aufgrund dieses Versprechens ließen sich über eine Million schwarzer Wähler im Süden des Landes registrieren. Es war also für beide großen Parteien, Demokraten und Republikaner, wichtig sich für Gerechtigkeit und Gleichbehandlung einzusetzen (Salvatore, 2007, s. 47).

Erst nachdem Kennedy von den Demokraten zu ihrem Kandidaten in der Präsidentschaftswahl ernannt worden war, erklärte er die Unterstützung seiner Partei für die Bürgerrechtsbewegungen und ließ sich von seinen afroamerikanischen Beratern über die Rassenprobleme, Ziele der Bürgerrechtsbewegungen und die Forderungen der Schwarzen informieren (Presler, 1984, s. 67). Den überwältigenden Erfolg heimste Kennedy ein, als M.L. King am 17. April 1960 ins Gefängnis gebracht wurde. John Kennedy kontaktierte Mrs. King telefonisch und teilte ihr seine Anteilnahme mit. Kennedy überredete den Richter, M. L. King gegen eine Kaution freizulassen (Nelson, 2000, s. 85). Nach der Freilassung von M.L. King überstimmte Kennedy seine Konkurrenten mit einer Mehrheit, von der $80 \%$ den Stimmen schwarzer Wähler zu verdanken waren.

Am Beginn verhielt sich Kennedy passiv zu den Bürgerrechtsbewegungen. Die Proteste gingen weiter und Kennedy verhielt sich apathisch verglichen mit den Erwartungen seiner schwarzen Wählerschaft. Er strebte eine Durchführung der Maßnahmen lediglich in jenen Bereichen, in denen die Bundesregierung bereits unumschränkte und unangefochtene Autorität besaß, an (Moosbrugger, 2006, s. 77). 19601961 konnte Kennedy keine Erfolge verzeichnen. Die harte Diskriminierung war noch an der Tagesordnung und die Regierung kam nicht voran. Wohnfrage, gerechte Beschäftigung und freier Zugang zum Arbeitsmarkt blieben ungelöste Probleme der Regierung (Oates, 1984, s. 92).

\subsection{Freiheitsfahrten-Freedom Riders}

1946 hatte der Oberste Gerichtshof das Verbot von Inlandsreisen für Farbige bereits aufgehoben. Aber die Aufhebung des Verbots wurde im Süden immer noch ignoriert. In diesem Zusammenhang regte der CORE im Mai 1961 eine sogenannte Freiheitsfahrt im ganzen Süden an. Nach der Planung fuhren zwei Busse mit Schwarzen und Weißen durch die südlichen Städte von Washington bis Jackson, Mississippi. Aber auf dem Weg nach Alabama wurden sie von Rassisten angegriffen und blutig Anprallen. Ein Bus wurde in Brand gesetzt. Die Insassen konnten sich nur mit Mühe aus den Flammen retten. Der 
zweite Bus wurde in Alabama gestoppt und die Passagiere zusammengeschlagen. Diese Begebenheiten führten wie erwartet zur Festnahme der Busfahrer.

In Montgomery wurden die Aktivisten am Busterminal von tausend Weißen niederknüppelt und schwer misshandelt. Nachdem diese Fälle weltweit in den Medien übertragen worden waren, beschlossen M.L. King und seine Freunde die Freedom Riders fortzusetzen. Die Regierung war von diesen Reisen nicht besonders erfreut; Kennedy wollte derartigen Aufruhr in den südlichen Städten verhindern, da die Vertreter der Südstaaten im Kongress viel Macht besaßen (Nelson, 2000, s. 86). Aber andererseits ging es um das internationale Image Amerikas und Kennedy konnte diese andauernde Missachtung von nationalen Gesetzen nicht länger tolerieren und musste einschreiten (Moosbrugger, 2006, s. 83). Deshalb schickte die Regierung eine Bundestruppe zum Schutz der Bürgerrechtler.

Die Freiheitsfahrten hatten letzten Endes Erfolg. Auf Robert Kennedys Anordnung hin erließ die Interstate Commerce Commission im September eine Verordnung, die der Rassentrennung in den Bahnhöfen der Überlandbusse ein Ende setzte (Critchlow, 2020, s. 84). Die Verordnung sollte am 1. November in Kraft treten. King bezeichnete sie als „einen bemerkenswerten Sieg“ (Oates, 1984, s. 218).

\subsection{Albany Rückschlag}

1962 kam es in Albany zu Protesten gegen die Rassentrennung und für die Wahlrechtsregistrierung. Die Polizisten waren gegen die Demonstranten unnachgiebig. Die Stadtbehörde wies die Forderungen der Schwarzen zurück und ließ die Demonstranten verhaften. Unter diesen Umständen versuchte die SNCC unter der Leitung von Martin Luther King mit Hilfe der örtlichen Bürgerrechtler gruppe in Albany eine Protestbewegung zu organisieren. Doch unter den Bürgerrechtlern herrschte Uneinigkeit über die Art und Weise der Proteste. Die Bürgerrechtler konnten sich nicht auf ihre gemeinsamen Ziele konzentrieren. Während sich die einen mit der Aufhebung der Segregation in öffentlichen Linien zufrieden geben wollten, wollten die anderen durch die Demonstrationen und Sit-in Aktionen die Integration in allen öffentlichen Einrichtungen und Geschäften der Stadt erreichen. Die Haltung der Polizei unter Polizeichef Pritschett war gegen die schwarzen Demonstranten freundlich und gewaltlos. Polizeichef Pritschett verbot den Polizisten Gewalt gegen die Demonstranten anzuwenden. Das war mit einem Grunde, dass die Bürgerrechtsaktionen zu keinem Ergebnis führten.

M.L. King verordnete einem der Afroamerikaner aufgrund des Bruchs der Gewaltfreiheit einen „Tag der Buße“ (Nelson, 2000, s. 88). Er und seien Freunde wurden daraufhin inhaftiert, was Spannungen innerhalb der schwarzen Gemeinde erzeugte. Manche Gruppen waren dafür es bei Aktionen auf lokaler Ebene zu belassen, andere waren dagegen. Schließlich verkündeten örtliche Bürgerrechtler, dass eine Vereinbarung mit der Stadtverwaltung erzielt worden sei (Moosbrugger, 2006, s. 85). Nach seiner Freilassung gegen ein Bußgeld erkannte M.L. King, dass es keine Möglichkeit mehr gab positiv auf die sich täglich verhärtenden Fronten einzuwirken (Nelson, 2000, s. 88), worauf er die Stadt verließ. Martin Luther King entwickelte neue Strategien. Er legte nun mehr Gewicht auf Einheit und Interdisziplinarität bei den Aktionen als früher.

\subsection{Sieg in Birmingham}

\section{FREE-DOM! (Slogan der Kinder beim Marsch auf Brimingham)}

M.L. King und die SNCC beschlossen nach der Niederlage in Alabama eine neue Strategie, die die Bürgerrechtsbewegung zum Erfolg führen würde. Die Bürgerrechtsbewegung entwickelte sich jedoch gerade in den Orten, wo die stärkste 
Segregation herrschte. Deshalb wurde Birmingham oder das sogenannte „Bombingham“ als idealer Schauplatz ausgewählt. Birmingham war immer stolz darauf, als die „am meistens segregierte Stadt Amerikas “zu gelten. Der Polizeichef Eugene „Bull“ Cannor war ein extremer Rassist und Freund des Ku Klux Klans. Die Bürgerrechtler waren hier auch aktiv unter dem Namen Alabama Christian Movement for Human Rights (ACMHR), aber ihre Forderungen, wie die Desegration des öffentlichen Verkehrs, der Restaurants, Toiletten und Trinkbrunnen in den Geschäften wurden unnachgiebig abgelehnt, die Bürgerrechtler verprügelt und vom Polizeichef ins Gefängnis gesteckt. Der weiße Mob verübte Bombenanschläge im Negerghetto.

Diesmal war die SNCC schon nach einem Monat bereit für einen neuen Einsatz. M.L.King schrieb einen offenen Brief, den berühmten „Letter from a Birmingham Jail“ an weiße Geistliche in Alabama, die ihn als unruhestiftenden Außenseiter bezeichneten, der nur Ärger nach Birmingham brächte und endlich einsehen sollte, dass die Zeit einfach noch nicht reif sei für Integration. (Nelson, 2000, s. 92).In dem Brief erklärte King, warum sie nicht mehr warten könnten und warnte davor, dass die Afroamerikaner eines Tages die Geduld verlieren könnten. Der Brief erregte großes Interesse bei den Bürgerrechtlern und in den Medien. Die Zeitungen druckten den ganzen Text ab. Nach der Entlassung M.L. Kings weigerte die Stadtverfassung sich die Einrichtungen zu verweigern. Bei jeder Demonstration wurden hunderte Demonstranten verprügelt und verhaftet. „Bull“ Cannor setzte das extrem brutale Vorgehen gegen die Protestierenden mit Hunden und Wasserwerfern fort.

Aufgrund der Not der Familien der Demonstranten und der geringen Zahl erwachsener Teilnehmer lösten sich die Demonstrationen schließlich auf. Doch M. L. King und die SCLC entschieden sich mit den Jungen bzw. Kindern weiter zu marschieren. Es war eine schwierige Entscheidung für M.L. King, weil sie damit ein großes Risiko eingingen und feindselige Kritik von allen Seiten kommen könnte (Oates, 1984, s. 281). Die Kinder könnten verletzt werden oder noch Schlimmeres könnte ihnen zustoßen. Andererseits aber wurden die schwarzen Kinder jeden Tag ihres Lebens durch die Diskriminierung verletzt. Am ersten Mai wurde der erste Marsch der Kinder festgesetzt. Am nächsten Tag marschierten über tausend Kinder durch die Straßen und sangen. Der Polizeichef „Bull“ Cannor befahl den Polizisten „die kleinen Nigger sofort einzusperren“ (Nelson, 2000, s. 95). Als das Ereignis in den Medien erschien, wirkte es wie ein Schock auf der ganzen Welt und auch in Amerika selbst. Bilder von Demonstranten, die von der Polizei brutal zusammengeschlagen wurden, Bilder von Jugendlichen, auf die Polizeihunde gehetzt wurden und von Kindern, die von Wasserwerfern getroffen gegen Wände geschleudert wurden (Nelson, 2000, s. 96).

Nach diesem Skandal wurde die Rassentrennungsverordnung in Birmingham (Desegregation des öffentlichen Lebens) schritt für schritt aufgehoben. Die Kennedys Regierung setzte sich von nun an voll für die Gleichberechtigung der afroamerikanischen Bevölkerung der USA ein (Berg, 2000, s. 395-397; Moosbrugger, 2006, s. 92).

\subsection{Marsch auf Washington}

"I say to you today, my friends, so even though we face the difficulties of today and tomorrow, I still have a dream. It is a dream deeply rooted in the American dream. I have a dream that one day this nation will rise up and live out the true meaning of its creed: "We hold these truths to be self-evident: that all men are created equal". ${ }^{2}$

\footnotetext{
2 Aus der berühmten Rede von Martin Luther King- Washington March
} 
Doch auch nach dem Sieg in Birmingham hörten die Rassenunruhen in den südlichen Städten nicht auf. Gleichzeitig gingen die Demonstrationen gegen die Rassendiskriminierung weiter. Die Regierung konnte sie nicht länger ignorieren. In einer Fernsehansprache trat John F. Kennedy die Verabschiedung eines Gesetzes zur Stärkung der Wählerrechte ein um die Rassendiskriminierung abzuschaffen (Moosbrugger, 2004, s. 98). Die Hauptziele waren im Regierungsprogramm die Aufhebung der Rassendiskriminierung, Verbesserung der wirtschaftlichen Situation der Schwarzen, Zugang zu öffentlichen Plätzen usw. festgesetzt. Doch leider blieb es nur beim Programm. In die Praxis wurden diese Ziele noch nicht umgesetzt. Zwar brachte Kennedy im Parlament immer wieder diese Forderungen, aber sie wurden immer vom Repräsententenhaus oder vom Senat aufgeschoben (Presler, 1984, s. 91). Was schließlich den Faden der Geduld zum Reißen brachte, war die Ermordung des geschäftsführenden Sekretärs der NAACP. Dieses Ereignis veranlasste M.L. King und seine Mitarbeiter eine neue Demonstration zu organisieren, aber diesmal mit zahlreichen Teilnehmern und großem Effekt.

Am 28. August 1963 bildeten die Demonstranten in Washington eine große Veranstaltung. Fast 200.000 Menschen, darunter ungefähr 60.000 Weiße, aus allen Teilen Amerikas fluteten nach Washington. Die Demonstranten versammelten sich friedlich am Washington Monument und marschierten singend zum Lincoln Memorial, das großen symbolischen Wert besa $\beta$, da es an die Freiheit als Grundrecht aller Menschen erinnert (Santoli und Vitulli, 2012, s. 7).

Martin Luther King hielt bei diesem Marsch eine Ansprache, die unter dem Titel „I have a Dream" weltweit berühmt wurde. In der Ansprache erklärte er, dass die Forderungen der Bürgerrechtsbewegung elementarer Bestandteil der demokratischen amerikanischen Tradition seien und die Weißen die Forderungen deshalb erfüllen müssten. Er forderte King die Schwarzen auf, weiterhin den Weg der Gewaltlosigkeit zu gehen und zusammenzuarbeiten (Nelson, 2000, s. 102) Die Regierung, bzw. John Kennedy gab seine Unterstützung für diesen Marsch und war sehr zufrieden mit dieser Aktion und Folgen. Nach dem Marsch hatten die Schwarzen endlich die Unterstützung der vielen liberalen Demokraten und einige kleine Rechte, sowie die Erhaltung des Demonstrationsgesetzes und der Schutz der Beamten vor Lynchensjustiz.

Nach dem Kennedys Ermordung erschütterten die Schwarzen in tief, weil sie große Unterstützer der Bewegung verloren hatten und die Bürgerrechtsgesetze, bzw. das Wahlgesetz ins Gefahr fallen könnte. Aber Präsident Lyndon Johnson erklärte gleich seine Unterstützung für die Bürgerrechtsbewegungen. Schließlich am 10. Februar 1964 verabschiedete das Repräsentantenhaus mit großer Mehrheit von 290 gegen 130 Stimme das Bürgerrechtsgesetz und daher mit der öffentlichen Unterstützung des Mehrheitsführers im Senat, Everett Dirksen, entschied sich der Senat am 10. Juni 1964 mit 71 zu 29 Stimmen „für eine Beendigung der Verschleppungspolitik“ und machte am 2. Juli den Entwurf gesetzeswirksam (Moosbrugger, 2006, s. 102).

\subsection{Das Freedom Sommer Projekt}

Mit der Verabschiedung des Bürgerrechtsakts steigern die Hoffnungen für Rassenruhe in Süden. Aber in kurzer Zeit sah man es, dass es Konflikt zwischen Segregationisten und Schwarzen noch gibt und vom Front $\mathrm{Ku}$ Klux Klan wurde die Stimme für die Verhinderung aller ethnischen Gruppen laut. Zum Beispiel; Während SNCC und SCLC im Süden seit 1962 die Kampagne der Wählerregistrierung durchführten, wurden dreiundsechzig Schwarzen ermordet. Mit dieser Bilanz erschienen die Aussagen Malcolm X, der immer extrem gegen der Bewegung den Hass und Stolz darstellte, für die 
Afroamerikaner noch glaubwürdiger. In diesem Zeitabschnitt wurden die Bürgerrechtler auf Zielscheibe von Rassisten gesetzt. Neun Bürgerrechtler, wobei zwei von ihnen Weiße waren, wurden getötet, viele Kirchen wurden verbrannt, viele Häuser waren durch Bomben zerstört (Moosbrugger, 2006, s. 122). Mit dem Beginn der Präsidentschaftswahl war dieses Projekt beendet.

\subsection{Von Selma nach Birmingham-1965}

We shall overcome! (Slogan der Bürgerrechtsbewegung)

In Selma, Alabama wohnte eine große afroamerikanische Bevölkerungsminderheit und darunter gab es viele potenzielle Wähler. Aber die Oberschicht von Dallas entschied es den Afroamerikanern das Wahlrecht vorzuenthalten und es wurde gleich in Selma durch den rassistischen und gewalttätigen Polizeichef durchgeführt. King erlebte mit der Regierung, und zwar mit FBI große Probleme. FBI Chef Edgar Hoover beschuldigte King eines Kommunisten, eines Heuchlers zu sein. Gleichzeitig lehnte der Präsident Johnson seine Unterstützung für die neue Wahlrechtsgesetzesvorlage ab (Nelson, 2000, s. 128).

All diese Entwicklungen führten King dazu einen neuen Marsch zu organisieren. Mit diesem Marsch hoffte er die öffentliche Beachtungen auf dieses Thema durch die Medien konzentrieren zu lassen. Der erste Protestmarsch begann 18. Januar 1965. Alle Alternativen wurden schon eingerechnet, Verhaftungen, Verprügeleien, alle gewalttätigen Bewegungen von der Gegnerseite. Die Proteste waren weiter und nächste Adresse für den nächsten Protest war der Marsch von Selma nach Montgomery. Mit der Regierungstruppen marschierten hunderten Demonstranten und daneben fünfzigtausend Anhänger im gesamten Staatsgebiet. Bei dem zweiten Protest lief wieder Polizeiunruhen, aber es brachte dabei für die Bürgerrechtsbewegungen ein Erfolg mit. Der sogenannte „Bloody Sonday“ Protest rief die Reaktion in der Öffentlichkeit hervor. Er wurde von amerikanischer Öffentlichkeit mit Erschrocken und Wut reagiert. „Die amerikanische Öffentlichkeit zeigte sich also zusehends unwillig die Misshandlung friedlicher schwarzer Demonstranten in Selma weiterhin hinzunehmen (Moosbrugger, 2006, s. 117).

Bis 15. März wurden viele Demonstranten, darunter ein weißer Pfarrer, getötet, viele wie immer ins Gefängnis geworfen. Letztlich erklärte Präsident Johnson erwartetes Wahlrechtgesetz in den Kongress einzubringen (Nelson, 2000, s. 129) „Ihre Sache muss auch unsere Sache werden" ruft er in einer leidenschaftlichen Rede. Er schließt mit den Worten der Bürgerrechtshymne: „We shall overcome“ (Bahr, 2004, s. 81).

Am 6. August 1965 wurde im Kongress die Voting Rights Act verabschiedet. Nach diesem Gesetz verbot alle Arten von Wahlsteuern, eben Test und Sonderregelungen, die jahrzehntelang zur politischen Entmündung der Schwarzen im Süden beigetragen hatten. Mit diesem Gesetz stiegen Wahlregistrierungen in südlichen Städten höher. Allein in Selma stieg die Wahlregistrierungszahl innerhalb von zwei Monaten (Wilk, 2020, s. 169). Daneben verbesserte dieses Gesetz auch das Schulsystem. Das Gesetz "education Act“ ermöglichte die Integration der Schwarzen Schüler in den weißen Schulen. Sie konnten endlich mit ihren Altersgenossen in gleichen Klasse ausgebildet werden.

Das Jahr 1965 war die Spitze der Bürgerrechtbewegungen. Die Bürgerrechtler bekamen in diesem Jahr die Unterstützung von allen Seiten der amerikanischen Gesellschaft und eben von der Regierung. Danach fanden sie nie wieder gleiche Interesse.

\subsection{Abflauen und Niedergang}

Schlechte Arbeitsmöglichkeiten, Diskriminierungen bei den Wohnmieten, schlechtes Ausbildungssytem und Verweigerung der Schwarzen am alltäglichen Leben 
verwandelten die optimistischen Hoffnungen im pessimistischen Zustand. Diese Umstände bewirkten bei King und seinen Mitarbeiter neue Hoffnungen.

Sie entschließen es, dass die Strategie des gewaltlosen Widerstandes für diese Probleme eine Lösung sei. Diese waren dagegen bereit die Bürgerrechtsbewegung auch in den Norden des Landes zu tragen und meinten, die SCLC sollte ihre Aktivitäten besser auf weitere Wählerregistrierungen im Süden konzentrieren (Moosbrugger, 2006, s. 144). Mit diesem Zweck hat King Chicago ausgewählt. 1966 zog er mit seiner Familie in Chicago ein, und mietete im Neger-Getto eine Wohnung, um seine Solidarität mit den Ärmsten der Armen zu beweisen (Presler, 1984, s. 115).

Es ist ihm gelungen, manche gewalttätige Jungen zu gewinnen. Im gleichen Jahr wurde in Chicago die Demonstrationen „Chicago Freedom Movement" und die „Campaign to End Slums“" begonnen. In Chicago begannen die Demonstrationen am 10. Juli 1966. 50.000 Menschen nahmen teil. Martin Luther King zeichnete sich in Demonstrationen radikal und harte Tendenzen ab und seine Äußerungen war immer ähnlich mit Malcolm X's. Nach der Demonstration legte eine Abordnung Vorschläge für bessere Schulen, bessere Arbeitsverhältnisse, bessere Wohnraumversorgung vor. Der Bürgermeister lehnte alle Vorschläge $a b$. Bürgermeister Richard Daley beschuldigte King, er würde nur Unzufriedenheit nach Chicago bringen und die Menschen zum Rassenhass aufstacheln (Nelson, 2000, s. 132).

Im Sommer kamen in Chicago schwere Unruhen heraus. Viele Geschäfte wurden geplündert. Die Verwaltung unterschrieb dabei ein Abkommen, in welchem sie sich verpflichtete Schwarzen zu menschenwürdigen Wohnungen zu verhelfen und die Ghettos aufzulösen (Presler, 1984, s. 118). Durch Medienberichterstattung wurde es bewiesen, dass es im Norden auch um die Rassentrennungen geht und es sehr weit verbreitet.

Die SCLC begann für die Vermeidung der Arbeitslosigkeit der Schwarzen mit der sogenannten „Operation Breadbasket“. Sie führte Boykottmaßnahmen gegen weiße Geschäfte, Läden und Märkte durch, die Schwarze benachteiligten oder es ablehnten, sie aufzunehmen. Dieser Boykott brachte eine gute Verbesserung im Arbeitsmarkt. Aber generell war die Chicagoer Kampagne in vielen Punkten erfolgslos. Kings Positionen stärkten immer nach der radikale Richtung.

\subsection{Vietnamkrieg- Armenfrage}

Wir müssen den Feuereifer der Bürgerrechtsbewegung mit der Friedensbewegung vereinen. Wir müssen demonstrieren, lehren und predigen, bis unsere Nation in ihren Grundfesten erschüttert ist (Oates, 1984, s. 508). Gegen den Vietnamkrieg wuchs ein kleiner Kreis, und ein Ring von diesem Kreis war Martin Luther King. Immerzu beschloss er seine Bürgerrechtsaktivitäten nur für den gewaltfreien Widerstand. King war immer gegen die Gewalt und die Gewaltarten gewesen. Mit Vietnamkrieg war er enttäuscht, weil er glaubte, dass sein Land als moralisch schlechtes Beispiel werde.

King sah den Vietnamkrieg als ,nichts vollbrachter Krieg“ (stanford.edu/King) und ein der grausamsten und sinnlosesten Kriege der Geschichte Martin Luther King führte 1967 eine Antikrieg-Marsch in Chicago mit der Angabe "The bombs in Vietnam explode at home - they destroy the dream and possibility for a decent America“.

King übte im Frühling 1967 in einem Friedensmeeting eine harte Kritik über die Ungerechtigkeiten des Krieges, Armutsfrage in USA, die mehre Beteiligung der schwarzen Soldaten als weißen an dem Vietnamkrieg und inner- außerpolitische Schäden der USA. King war schon überzeugt, dass der Krieg über die Armenkosten geführt wurde, das wesentliche Beispiel; mehr Einsatz der schwarzen Soldaten als die weißen auf dem 
Schlachtfeld. Mit solchen Angaben wurde er von FBI und Weißen, manchmal aber Schwarzen, als Kommunisten und Verräter genannt. Daher belastete Präsident Johnson bzw. Weißhaus King als nicht gern gesehene Person und die Beziehung zwischen Weißhaus und Bürgerrechtsbewegung verschlechterte sich (Presler, 1984, s. 121).

Martin Luther King bedauerte die scharfe Rede über die Armutsfrage und den Vietnamkrieg bis seinem Tod am 4. April 1968. Er verzichtete niemals gewaltslose Methoden während seinen Kritiken und Aktionen.

\subsection{Kings Mord}

In einer Rede verdeutlichte King seine Todesahnung ganz klar. King nahm wie immer an einer Demonstration für Müllarbeitstreik im Memphis teil. Nach seiner Rede versprach er, am 8. April noch eine Demonstration durchzuführen. Er hielt vor einem Tag seines Todes die letzte Predigt. In dieser Predigt ging es um den Glauben an der Gewaltlosigkeit und der Gemeinsamkeit.

Am 4. April 1965, Memphis, Tennessee. Alle Vorbereitungen für den Marsch waren einwandfrei. Er überredete die jungen radikalen Schwarzen für die Teilnahme an dem Marsch. Das Demonstrationsverbot wurde von dem Gericht aufgehoben. Martin Luther King war damit zufrieden (Nelson, 2000, s. 145). Er war ausgesprochen guter Laune. Als Martin Luther King und seine Freunde zum Abfahrt auf dem Balkon warteten, wurde er am Kopf erschossen. Er wurde gleich ins Krankenhaus gebracht, aber er starb im Krankenhaus um 19:05 Uhr. Er war - genau wie Malcolm X zum Zeitpunkt seines Todes 39 Jahre alt. Als Kings Mörder wurde James Earl Ray, der ein Rassist und ehemaligen Häftling war, verhaftet. Es gab viele Morddrohungen gegen King. Beispielsweise, der Ku Klux Klan setzte ein Kopfgeld für die Ermordung Kings aus und bis 1968 langte an die fünfzig Morddrohungen beim FBI ein (Moosbrugger, 2006, s. 158). Nach dem Tod Kings kam es in vielen Städten zu Unruhen. In den zwei Tagen wurde 49 Menschen, meistens Schwarze, getötet und viele verhaftet. Zur Beruhigung der Situation ließ Präsident Johnson die Fahnen auf Halbmast setzen und erklärte Sonntag, den 7. April 1968 zum nationalen Trauertag. Mit dem Tod Martin Luther King, sowie auch Malcolm X rückte „das Traumziel von einer gerechten Gesellschaft, einer Gesellschaft der verwirklichten Bürgerund Menschenrechte“ in weiter Ferne.

\section{Theoriebezogene Rahmen}

In diesem Teil wird es auf die Nachrichtenfaktoren und die verwandte Ansätze eingegangen.

\subsection{Nachrichtenwert-Theorie}

Unter der Nachrichtenwert- Theorie versteht man die Nachrichtenauswahl sowohl aus theoretischer Perspektive als auch aus der Perspektive des praktischen Journalismus thematisierten und zudem eine Reihe von Auswahlkriterien (Eilders, 1997, s. 19). In der Theorie werden die Realitätskriterien und die Grundformulierungen der Nachrichten diskutiert. Die Theorie zeigt, dass die Vorstellung der Wirklichkeit in den Medien nicht nur aufbauen kann. Die Wirklichkeit kann nur als mediale Konstruktion vor dem Hintergrund der Forschungstraditionen wie Nachrichtenwert-Theorie hinreichend argumentativ begründet werden (Burkart, 2002, s. 283).

Der Grundbau der Nachrichtenwert- Theorie wurde zuerst von Walter Lippmann dargestellt. Nach Lippmann bildet die Nachrichtenwert die Publikationswürdigkeit von Ereignissen, die sich aus dem Vorhandensein und der Kombination verschiedener Ereignisaspekte ergibt. Daneben legte Lippmann jede Auswahltätigkeit auch eine 
Interpretation dar. Er sieht individuelle und kulturelle Stereotypen als Kriterien für die unterschiedlichen Interpretationen (Eilders, 1997, s. 71).

Die andere wichtige Vertreter ist Einar Östgaard, der die europäische Forschungstradition begründete. Laut Östgaard basiert das Forschungsinteresse darauf, dass die Verzerrungen im internationalen Nachrichtenfluss betrachtet werden. Er sieht vorerst Unterschiede zwischen externen Faktoren und internen Faktoren. Während es bei externen Faktoren um ,direkte oder indirekte Einflussnahmen von Regierungen, Nachrichtenagenturen und Eigentümern von Massenmedien aus politischen oder ökonomischen Interessen" geht, handelt es sich bei internen Nachrichtenfaktoren um einzelne Aspekte von Nachrichten, die diese für die Rezipienten interessant und beachtenswert machen (Staab, 1990, s. 56).

Daher hat der Forscher, um den Zeitungsinhalt festzulegen, drei Faktoren genannt. Diese Faktoren sind (Staab, 1990, s. 57);

- Vereinfachung; die komplexen Sachverhalten werden von den Journalisten so einfach wie möglich dargestellt. Das heißt, die komplexe Geschehensverhältnisse werden auf eine möglichst einfache Struktur reduziert.

- Unter Identifikation versteht man; dass die Nachrichtenmedien versuchen, die Aufmerksamkeit ihrer Rezipienten zu gewinnen, indem sie über die bekannten Ereignisse, Sachverhalten und Themen berichten.

Und Sensationalismus ist es, die Aufmerksamkeit ihrer Rezipienten zu gewinnen, indem man die Nachrichten so dramatisch wie nur möglich und auch emotional darstellt.

Letzte Äußerung der Nachrichtenfaktoren wird von Schulz gegeben. Bei der Auswahl der Nachrichtenfaktoren für die empirische Analyse ging Schulz pragmatisch mit den europäischen Studien zur Nachrichtenwert- Theorie vor. Er verwendete die Faktorenliste von Galtung- Ruge, die im Hinblick auf die erweiterte Fragestellung modifiziert wurde. Hauptkriterium für die Annahme eines Faktors in der Liste war dessen Operationalisierbarkeit (Eilders, 1997, s. 35).

Die Liste von Schulz besteht aus 6 Haupt Nachrichtenfaktoren und 13 Unterfaktoren. Diese sind: Dauer, Thematisierung, räumliche, politische und kulturelle Nähe, Relevanz, regionale und nationale Zentralität, persönlicher Einfluss, Prominenz, Überraschung, Komplexität, Konflikt, Kriminalität, Schaden, Erfolg, Personalisierung und Ethnozentrismus (Burkart, 1995).

1- Zeit, Dauer: Der Nachrichtenwert punktueller Ereignisse von kurzer Dauer ist höher als jener von Langzeitereignissen. Thematisierung: je etablierter ein Thema in der Berichterstattung ist, um so höher ist sein Nachrichtenwert.

\section{2- Nähe}

a. Räumliche Nähe: Ereignisse, die in geographischer Nähe zum Redaktionssitz liegen, werden eher Berücksichtigung finden.

b. Politische Nähe: Dasselbe gilt für die Ausprägung der wirtschaftspolitischen Beziehungen zum Ereignisland, die den Nachrichtenwert erhöhen.

c. Relevanz: Der Grad der Betroffenheit und existentiellen Bedeutung eines Ereignisses. 


\section{Status}

a. Regionale Zentralität: Der Grad der politisch-ökonomischen Bedeutung der Ereignisregion (bei nationalen Nachrichten)

b. Nationale Zentralität: Ausdruck für die wirtschaftliche, wissenschaftliche, und /oder militärische Macht des Ereignislandes (bei internationalen Nachrichten)

c. Persönlicher Einfluss: Politische Macht der involvierten Personen

d. Prominenz: Bekanntheitsgrad von Personen (bei unpolitischen Meldungen)

\section{Dynamik}

a. Überraschung: Erwartbarkeit des Zeitpunktes und Resultats eines Ereignisses

b. Struktur: Komplexität des Verlaufs, dessen Form, sowie Beteiligung und Überschaubarkeit des Ereignisses.

\section{Valenz}

a. Konflikt: Grad der Aggressivität verschiedener Ereignisse auf politischer, wirtschaftlicher oder kultureller Ebene. Kriminalität: die Rechtswidrigkeit von Handlungen,

b. Schaden: die Personen-, Sach- und finanziellen Schäden, und Erfolg: die Fortschritte auf politischem, wirtschaftlichem oder kulturellem Gebiet, die die Ereignis bewirkt (Staab, 1990, s. 82).

\section{Identifikation}

a. Personalisierung: Grad des persönlichen Bezugs zum Ereignis

b. Ethnozentrismus: Betroffenheit der Bevölkerung des Landes, in dem jeweiligen Medium erscheint.

Später überarbeitete Schulz den Katalog der Nachrichtenfaktoren. Damit hatte er zwei Ziele: Eins von denen war es, „Faktoren, die mehrere Dimensionen enthielten, präziser zu fassen, und die Faktoren, die nach Ansicht des Autors in ungerechtfertigter Weise differenziert worden waren, globaler zu definieren“ (Staab, 1990, s. 86).

\section{Empirie bezogener Rahmen}

In diesem Teil wird das bereits im ersten Teil erläutertes Thema über die Bürgerrechtsbewegungen 1956-1968 und die aufgestellte Hypothesen, durch „Arbeiter Zeitung “ überprüft.

\subsection{Untersuchungsmethode: Quantitative Inhaltsanalyse}

Um die Forschungsfrage und die Hypothesen $\mathrm{zu}$ erleuchten, werden die Berichterstattungen mit der Hilfe der Methode "Quantitativen Inhaltsanalyse“ durchgeführt. Die Inhaltsanalyse befasst sich mit Kommunikationsinhalten, die durch die Forschungsfrage eingegrenzt ist. Sie bezieht sich außerdem auch auf Analyseeinheiten, an denen diese Kommunikationsinhalte gemessen werden (Rösler, 2005, s. 82). Und auch ist die Inhaltsanalyse als eine empirische Methode zur systematischen, intersubjektiv nachvollziehbaren Beschreibung inhaltlicher und formaler Merkmale von Mitteilungen zu verstehen (Früh, 2007, s. 21).

\subsection{Untersuchte Zeitung und Untersuchungszeitraum}

Da es in der Arbeit offen darlegen werden sollte, wie die Bürgerrechtsbewegungen in USA und die Hauptpersonen in den österreichischen Medien bzw. Zeitungen behandelt 
wurden, wird eine österreichische Zeitung und zwar die „Arbeiter Zeitung “ ausgewählt. Die Arbeiter Zeitung wurde erstmal 1886 gegründet und von Victor Adler herausgegeben. Nachdem das Blatt 1889 verboten wurde, gründete Adler am gleichen Ort die ArbeiterZeitung, die erstmals am 12. Juli 1889 erschien, zuerst zweimal im Monat, ab 18. Oktober 1889 wöchentlich, und ab 1. Januar 1895 täglich - bis zum Verbot am 12. Februar 1934.

In der Arbeit wurde der Untersuchungszeitraum nach der Chronologie der Bürgerrechtsbewegungen festgestellt. Der gesamte Untersuchungsraum umfasst alle Ausgaben der Arbeiter Zeitung vom Jahr 1956 (Sieg beim Montgomery Busboykott) bis 1968 (Mord Martin Luther Kings).

Bis zum Jahr 1963 hat sich die Arbeiter Zeitung in fast keinem Artikel mit den Bürgerrechtsbewegungen in USA befasst. Allgemein wurden in diesem Zeitraum die Mitgliedschaft bzw. Aufnahme Österreichs in die Vereinigten Nationen, die heftige Ungarnfrage bzw. die Arbeiterbewegung in Budapest sowie die kommunistischen Versuche in Europa behandelt. Und nur ein Artikel über den Sieg vom „Montgomery Busboykott" wurde veröffentlicht. Zwischen den Jahren 1963-1968 wurden die Ereignisse parallel zur Chronologie regelmäßig in der Zeitung feststellt und analysiert. Insgesamt wurden 118 Artikel mit Kommentaren und Berichten gelesen und analysiert.

\subsection{Nachrichtenfaktoren und ihre Auswertungen}

Als Nachrichtenfaktoren wurden zu den+ Artikeln passende Faktoren ausgewählt und analysiert.

1. Etablierung

2. Dauer

3. Status der Ereignisnation

4. Politische Elite-Person

5. Nicht-politische Elite-Person (aus Sport, Kultur, Medien etc.)

6. Überraschung

7. Struktur/ Komplexität

8. Gewaltsamer Konflikt/ Kriminalität

9. Nicht Gewaltsamer Konflikt

10. Schaden

11. Erfolg

12. Personalisierung

13. Ethnozentrismus

14. Demonstration

\subsection{Hypothesen und Fragestellungen}

Folgende Forschungsfrage und Hypothesen ergaben sich als forschungsleitend:

\section{Forschungsfrage:}

Mit welchen Aspekten wurden die Bürgerrechtsbewegungen und die Hauptpersonen in der Arbeiter Zeitung dargestellt? 


\section{Hypothesen:}

1. In der Arbeiter Zeitung kommen die Nachrichtenfaktoren „Gewalt“, „Personalisierung“ und „Ethnozentrismus“ häufiger vor als die anderen Faktoren.

2. In Arbeiter Zeitung wird der Hauptgrund der Bürgerrechtsbewegungen relativ gering erläutert. Im Vordergrund stehen meistens die Demonstrationen, die Krawalle in den Südstaaten der USA und gewalttätige Aggressionen der Polizeibehörden während den Demonstrationen.

3. In Kommentaren wird, als das Problem der Schwarzen, nur Rassentrennung in der Gesellschaft wahrgenommen. Auf die anderen Probleme, wie die soziale, wirtschaftliche Lage der Schwarzen werden in den Kommentaren nicht mehr Rücksicht genommen.

\subsection{Interpretationen und Auswertungen der Fragestellung und Hypothesen}

In diesem Abschnitt wird die Fragestellung und die Hypothesen nach der Analyseergebnisse erklärt. Die Tabellen sollten drei Ebene gestellt werden, in der je nachdem Themenverteilung, Personenverteilung und Verteilung der Nachrichtenfaktoren kategorisiert wurde. Die Forschungsfrage wurde nach diesen Tabellen interpretiert.

\subsubsection{Auswertungen der Forschungsfrage}

Mit welchen Aspekten wurden die Bürgerrechtsbewegungen und die Hauptpersonen in der Arbeiter Zeitung dargestellt?

Tabelle 1. Themenverteilung in den untersuchten Artikeln

\begin{tabular}{|l|c|c|}
\hline Themen & A: $\mathbf{1 2 0}$ & \% Häufigkeit \\
\hline Kriminalität & 132 & 1,10 \\
\hline Wirtschaftlich & 34 & 0,28 \\
\hline Politisch & 32 & 0,27 \\
\hline Sozial- gesellschaftlich & 24 & 0,20 \\
\hline Militärisch & 13 & 0,11 \\
\hline Sonstige & 6 & 0,05 \\
\hline
\end{tabular}

Hier wurde insgesamt 120 Artikel untersucht. Aus diesen Häufigkeitsverteilungen lässt sich schließen, dass die Arbeiter Zeitung ihre Berichterstattung stark auf die kriminelle, politische, und sozial-gesellschaftliche Aspekte konzentriert hat. Wirtschaftliche Aspekte wurden in ihren Berichterstattungen kaum behandelt.

Im Rahmen der Kriminalität 1,10\% kommen die gewalttätigen und gewaltlosen Aktionen der Bürgerrechtler, Demonstrationen in den Bundesstaaten bzw. südlichen Staaten, Krawalle zwischen Polizei und den Demonstranten, Verbrechen, Rassenunruhen in den Städten, Schaden während des Plündern, sowie (der Demonstrationen und) Verhaftungen vor.

Was das Wirtschaftliche angeht, werden 0,28 Prozent die ökonomischen Schäden, wie wirtschaftliche Probleme von beiden Gesellschaften, die enorme Arbeitslosigkeit, Rassendiskriminierung an den Arbeitsplätzen, Erhöhung der Wohnmiete und die Armutsfrage berücksichtigt.

Im politischem Bereich, das 0,27 Prozent ausmacht, wurde häufig die Annäherung der Regierung und Senat zur Abscheidung der Bürgerrechtsgesetze, die Durchführungsproblem der Gesetzentscheidungen in der Gesellschaft und die Problemen 
bei denen gewaltlose und gewaltsame Problemen zwischen Schwarzen und Bundesbehörde, bzw. zwischen Bürgerrechtführer und die Gouverneur behandelt.

Im Sozial-gesellschaftlichen Bereich, 0,20 Prozent, werden die Rassentrennungen in der amerikanischen Gesellschaft, die Vereinigung der Weißen und Schwarzen, das besondere Bildungssystem für die Schwarzen, die Unterstützung und Betrachtung der Gesellschaft gegenüber Schwarze und die demografische Darstellung der Schwarzen im Süden und Norden und deren Ursachen berücksichtigt.

Im militärischen Bereich, 0,11 Prozent, geht es um den Einsatz der militärischen Kräfte in den Konflikten in verschiedenen Orten und Intervention der Regierung durch die Bundestruppen. Aber auch um die Aufstände in den Städten und Sonderfälle in der Bundesbehörde, da manche Bundesbehörden die Durchführung der Beschränkung von Rassendiskriminierungen verweigerten und zudem verhinderten.

Tabelle 2. Personenverteilung in den untersuchten Artikeln

\begin{tabular}{|l|c|c|}
\hline Personen & A: 120 & \% Häufigkeit \\
\hline Martin Luther King & 37 & 0,31 \\
\hline Lundon Johnson & 14 & 0,12 \\
\hline John F.Kennedy & 10 & 0,08 \\
\hline Robert Kennedy & 5 & 0,04 \\
\hline Malcolm X & 3 & 0,03 \\
\hline
\end{tabular}

Bei der Untersuchung der Personenverteilung zeigt sich, dass Martin Luther King die Hauptfigur in der Berichterstattung war und zwar mit 31 Prozent.

In den Artikeln wird Martin Luther King (0,31 Prozent), mit der Rolle als Bürgerrechtsführer und Organisator der Demonstrationen, der Apostel der gewaltlosen Bewegung in den USA, der Gläubiger Pfarrer sowie einen Friedensnobelpreis tragender schwarzer Mann in den Vordergrund gerückt. Das private Leben Kings wurde in den Artikeln nie darlegt.

Der amerikanische Präsident Lundon Johson, 0,12 Prozent, zeigt sich in den Berichten als ein Mann mit gutem Kontakt zur schwarzen Bevölkerung, jedoch auf eine sehr maßvolle Art. Und außerdem mit seinen Anstrengungen zur Verabschiedung der Bürgerrechtsgesetze im Senat und Kongresshaus.

Der Präsident John F. Kennedy, 0,08 Prozent, wurde in den Berichten als Negerfreund dargestellt. Daneben wurden in den Artikeln seine Unterstützungen und seine Einsätze an Schwarze gegen die Rassentrennungen in Bundesbehörden sowie die Schulen, Universitäten mit Lob behandelt.

Der Justizminister Robert Kennedy, 0,08 Prozent, gleichzeitig auch der Bruder vom Präsident Kennedys, wurde mit seiner guten Laune gegen Schwarze und seine Toleranz für die Bürgerrechtsbewegungen erläutert.

Der andere Bürgerrechtsführer, Malcolm X, 0,03 Prozent, wurde in Artikeln als ein extremer und gewalttätiger Führer darstellt. 


\subsubsection{Auswertungen der Hypothesen}

Tabelle 3. Verteilung von Nachrichtenfaktoren in den Untersuchten Artikeln A-Z

\begin{tabular}{|l|c|c|}
\hline Nachrichtenfaktoren & A.120 & Häufigkeit\% \\
\hline Gewaltsamer Konflikt/Kriminalität & 51 & 0,43 \\
\hline Etablierung der Themen & 48 & 0,40 \\
\hline Ethnozentrismus & 44 & 0,37 \\
\hline Politische Elite Person & 38 & 0,32 \\
\hline Demonstration & 37 & 0,31 \\
\hline Status der Ereignisnation & 31 & 0,26 \\
\hline Schaden & 25 & 0,21 \\
\hline Dauer & 24 & 0,20 \\
\hline Personalisierung & 20 & 0,17 \\
\hline Nicht gewaltsamer Konflikt/Kriminalität & 19 & 0,16 \\
\hline Nicht politische Person & 16 & 0,13 \\
\hline Struktur/Komplexität & 15 & 0,13 \\
\hline Erfolg/Recht & 14 & 0,12 \\
\hline Überraschung & 3 & 0,03 \\
\hline
\end{tabular}

\section{Hypothese 1}

In der Arbeiter Zeitung kommen die Nachrichtenfaktoren „gewaltsamer Konflikt/Kriminalität“, „Personalisierung“ und „Ethnozentrismus“ häufiger vor als die anderen Faktoren.

Diese Hypothese muss verworfen werden, da der Faktor „Personalisierung“ mit einem Wert von 0,17 Prozent nicht so häufig erwähnt wird wie „Etablierung der Themen“, das satte 0,40 Prozent ausmacht. Man kann hier davon ausgehen, dass die sensationelle Seite der Ereignisse wie ,gewaltsamer Konflikt 0,43 Prozent oder „Demonstrationen 0,31 Prozent für die Arbeiter Zeitung bedeutsamer waren, als die Hintergrundkenntnisse wie die Personalisierung, nicht politischer Elite Person, 0,13 Prozent für die Zeitung ein Ereignis ist.

Der Nachrichtenfaktor „Ethnozentrismus“, 0,37\%, ist, wie auch die Personalisierung, nicht häufig erwähnt. Beim Nachrichtenfaktor Ethnozentrismus geht es um den Zusammenhang zwischen dem Hauptgrund der Bewegungen und der Rolle der Nation im Ereignis. Es scheint so, dass auf Grund der eigenen politischen Aspekte, die Arbeiter Zeitung die Situationen der Schwarzen in den Artikeln behandelte.

Der Nachrichten Faktor „Etablierung“ 0,40\%, wird in der Arbeiter Zeitung mehr als andere Nachrichtenfaktoren wie beispielsweise Schade, Erfolg, Dauer, Status der Ereignisnation etc. erläutert. Hauptursache hierfür sind, die führenden Probleme der schwarzen Bevölkerung, die inneren sozial-gesellschaftlichen Diskriminierungen, die schlechte wirtschaftliche Lage, sowie die Ausbildungsprobleme. Diese werden in den Kommentaren berichtet, ausgetauscht und diskutiert.

Politische Elite Person, 0,32\%, spielt in den Bürgerrechtsbewegungen in den USA eine gestaltende Rolle. Daher berichtet auch die Arbeiter Zeitung über die Struktur der Bürgerrechtsbewegungen durch die politischen Hauptpersonen. Die Arbeiter Zeitung führt 
durch die politische Person die gesetzlichen Erfolge wie Bürgerrechte, eben die militärischen Kräfte der Regierung und die Konflikt der zwischen Institutionen auch an.

\section{Hypothese 2}

„In den Berichten werden die Hauptgründe der Bürgerrechtsbewegungen relativ gering erläutert. Im Vordergrund stehen meistens die Demonstrationen, die Krawalle in den Südstaaten in USA und gewalttätige Aggressionen der Polizeibehörden in den Demonstrationen."

Die Hypothese muss beibehalten werden. Sowie es oben bereits dargestellt wurde, werden die Ereignisse in der Zeitung auf eine sensationelle Art und auch sehr ausführlich wiederspiegelt. Die Reaktionen und Aggressionen zwischen den Weißen und den Schwarzen überschatteten die Hauptzwecke der Bewegung. Aber gleichzeitig sollte berücksichtigt werden, dass die Arbeiter Zeitung die österreichische Leserschaft durch die emotionalen Berichte sensibilisieren wollte, die Rassentrennungen als ein Problem der ganzen Welt wahrzunehmen. Die Arbeiter Zeitung begann das Publikum zu informieren, nachdem die Bewegung einige (Wendepunktwerte) Fortschritte gemacht hatte. Diese Lücke wurde später mit den Kommentaren, Korrespondenten Berichten aus der Nähe des Geschehens beseitigt. Mit welchem Ereignis die Bürgerrechtsbewegung gestartet wurde, wurde in den Berichten am Ende der Aktion nur einmal dargestellt, und zwar nur das erfolgreiche Ergebnis als kurze Meldung.

Bis die Bewegung mit den Aktionen beschleunigt wurde, kamen die zwischen Ära in den Berichten nicht vor. Die darauf folgenden Berichten behandelten nicht diese Ära, z.B. Sit-in Bewegungen. Nur in den Geschichtsberichten oder in einigen Kommentaren wurden die einführenden Bemerkungen, kurze Geschichten der Bewegung und die Informationen über die wichtigen führenden Personen gegeben.

\section{Hypothese 3}

„In Kommentaren wurde als das Problem der Schwarzen nur Rassentrennung in der amerikanischen Gesellschaft wahrgenommen. Die anderen Probleme, sowie die soziale, wirtschaftliche Lage der Schwarzen wird in den Kommentaren nicht berücksichtigt.“

Diese Hypothese sollte weggeworfen werden, da in den Kommentaren das „Negerproblem“ nicht nur als bezüglich der Rassentrennung in der amerikanischen Gesellschaft wahrgenommen wurde, sondern es wurde als das Problem der ganzen Welt interpretiert. Daher werden auch in den Kommentaren häufig für führende Personen wie Martin Luther King und seine wertwürdige Stelle in den Bürgerrechtsbewegungen und in der schwarzen Gesellschaft Lob ausgesprochen. Seine guten Taten wie vor allem Widerstand ohne Gewalt in der wirtschaftliche Engpasse der Schwarzen, die Rassentrennungen des Farbigen, die Rassendiskriminierungen auf Arbeitsplätzen sowie Schulen, bei der Wohnmiete, rechtliche und gesellschaftliche Benachteiligungen der Schwarzen, gleichzeitig die politischen Entwicklungen der Afrikanischen Länder und die Stelle und Macht der Negerorganisationen, wurden immer wieder erwähnt. Daneben wird in den Kommentaren die Gewalttätigkeit von beiden Seiten, also Schwarzen und Weißen, scharf kritisiert und die Ursachen der gewaltsamen Aktionen meistens mit den ungerechten wirtschaftlichen und sozialen Verteilungen begründet.

Allgemein zeigt die Arbeiter Zeitung eine starke Tendenz auf eine sozialdemokratische Linie. Daher werden auch in Kommentaren genau aus diesem Grund selten die so genannten gefährlichen Einflüsse der politischen Strömungen, sowie die kommunistischen Strömungen eben wie die Studenten-Bewegungen in Europa behandelt und meist die Bürgerrechtsbewegungen im großem Rahmen analysiert. 


\section{Schlussbemerkung}

Schon in frühen Zeiten wurde das Thema über die Rassentrennungen in verschiedenen Gesellschaften heftig diskutiert und um eine sichere Lösung finden zu können wurden die verschiedensten Methoden entwickelt. Die Gewaltlose Bewegungen und Boykotte gegen die Diskriminierungen erwiesen sich als die stärksten Waffen. Heutzutage kommt es $\mathrm{zu}$ immer mehr Auseinandersetzungen wegen Rassendiskriminierungen in den vielen Ländern, bzw. vor allem in den westlichen Ländern. Die Auseinandersetzungen werden über die Medien mehr thematisiert als in anderen Vermittlungen wie Organisationen gegen rassistische Handlungen oder auch in offiziellen Behörden. Heute treten Medien an die Stelle der Gewaltlosen Methoden. An solchen spekulativen Ereignissen sind die Medien unerlässlich, das Publikum zu manipulieren und zu schaffen, dass die Rezipienten die Ereignisse in breitem Spektrum betrachten können. Der Rezipient ist aber dann bereit, die Ereignisse wahrzunehmen und zu handeln, wenn die behandelten Themen in den Berichterstattungen die Anpassungen der verschiedenen Darstellungsformen von der Wichtigkeit der Ereignisse abhängen.

Die Mission, die das Publikum den Medien aufträgt, erfüllen die Medien bzw. die Zeitungen mit den Berichterstattungen. Dieses unabdingbare Medium legt die Ereignisse in bestimmter Art und Weise mit den schon festgestellten Funktionen dar. Diese Kriterien erhöhen in einer Berichterstattung die Wirklichkeitswerte und dadurch beweisen sich die Zuverlässigkeiten der Ereignisse. Diese Kriterien, also die Nachrichtenfaktoren wurden in der Arbeit durch die Berichterstattung der Arbeiter Zeitung durchgeführt. In den Berichten lassen sich sowohl die Häufigkeiten der Faktoren als auch die Tendenzen des Inhalts der Berichte erkennen. Die angeführten Resultate durch die Faktoren bestätigen einerseits bestimmte Vorannahmen und Hypothesen und andererseits werden unpassende Hypothesen widerlegt.

Die Einschätzungen lauten wie folgt:

- Arbeiter Zeitung berichtete intensiv über die kriminellen Konflikte in den USA, die durch die Bürgerrechtsbewegungen ausgelöst wurden. Die amerikanische Regierung wollte einerseits die Bürgerrechtsgesetze verabschieden, aber andererseits nicht, weil es in mancher Umgebung unangenehm begrüßen werden könnte, wie beispielsweise im Senat und Kongresshaus.

- Kings gewaltloses Konzept wurde in den Berichten, bzw. Kommentaren mit Lob erwähnt. Im Allgemein wurden die Bürgerrechtsbewegungen für Bewegungen mit guten und wahren Aspekte gehalten.

- Gegenüber gewaltsamen Konflikten und auch Kriminalität zeigte die Arbeiter Zeitung eine sehr kritische Haltung. Erwähnenswert ist jedoch, dass interessanterweise, wenn es um gewalttätige Aktionen im Zusammenhang mit den Bürgerrechtlern ging, diese in den Berichten und auch Kommentaren immer gerechtfertigt wurden. Aber andererseits wurden die Gewalttätigen Aktionen in der Arbeiter Zeitung hart kritisiert.

Abschließend sollte man hier erwähnen, dass trotz der Bürgerrechtsbewegungen die innere Frage der USA, heute vor allem durch eine neue mediale Betrachtungsweise in Protesten und Demonstrationen ersetzt wurde bzw. sich entwickelt hat. In diesem Sinn ist es wichtig, welche Medien, wie und von welchen Aspekten aus, auf Bewegungen eingehen. In der Arbeiter Zeitung wurden zwar manche Aspekte wie die Hauptgründe der Bewegung, in den Berichten nicht genau erläutert, aber in den Kommentaren und in den Korrespondenten-Berichten, sowie Serienberichten wurden viele Betrachtungen mit 
verständlichen Hinweisen aufgezeigt. In diesem Zusammenhang kann die wirksame Waffe „gewaltlose Methode“ durch die Berichterstattungen ihre Macht darstellen, wie man ohne Gewalt seine eigenen Rechten schützen kann, auch wenn es manchmal sehr viel Kraft kostet.

\section{Quellenverzeichnis}

Bahr, H. E. (1990). Seht, da kommt der träumer. Unterwegs mit Martin Luther King. Stuttgart: Kreuz Verlag.

Bahr, H. E. (2004). Martin Luther King, für ein anderes Amerika. Berlin: TaschenbuchVerlag.

Berg, M. (2000). The ticket to freedom: die NAACP und das Wahlrecht der AfroAmerikaner. Campus Verlag.

Blumberg, R. L. (1984). Civil rights the 1960s freedom struggle; social movement past and present. Boston, Massachusetts: Twayne Publisher.

Burkart, R. (1995). Kommunikationswissenschaft. Grundlagen und problemfelder. Umrisse einer interdisziplinären sozialwissenschaft. Wien, Köln: Böhlau Verlag.

Burkart, R. (2002). Kommunikationswissenschaft. Köln, Weimar, Wien: Böhlau Verlag.

Butter, M., Franke, A. und Tonn, H. (Eds.). (2016). Von Selma bis Ferguson-Rasse und rassismus in den USA (Vol. 15). Transkript Verlag.

Critchlow, D. T. (2020). In defense of populism: Protest and American democracy. University of Pennsylvania Press.

Eilders, C. (1997). Nachrichtenfaktoren und rezeption; eine empirische analyse zur Auswahl und Verarbeitung politischer Information. Opladen: Westdeutscher Verlag.

Engel, M. (2006). The civil rights movement in the USA- from Rosa Parks to black power. Universität Wien: Diplomarbeit.

Früh, W. (2007). Inhaltsanalyse. Konstanz: UVK Verlaggesellschaft.

Moosbrugger, D. (2004). Die amerikanische Bürgerrechtsbewegung, „Schwarze Revolution " in den 1950er und 60er Jahren. Stuttgart: Ibidem- Verlag.

Nelson, W. B. (2000). Martin Luther King-Malcolm X Gegenspieler. Frankfurt am Main: Fischer Taschenbuch Verlag.

Newfield, J. (1988). Robert Kennedy: A memoir. New York: New American Library.

Oates, S. B. (1984). Martin Luther King; Kämpfer für Gewaltlosigkeit. Hamburg: Ernst Kabel Verlag.

Pressler, G. (1984). Martin Luther King. Hamburg: Rowohlt Taschenbuch Verlag.

Rösler, P. (2005). Inhaltsanalyse. Konstanz: UVK Verlaggesellschaft.

Salvatore, S. C. (2007). Civil rights in America: Racial voting rights-A national historic landmarks theme study. National historic landmarks program (Washington, DC: National Park Service, 2007, revised 2009).

Santoli, S. P. und Vitulli, P. (2012). Examining the 1963 March on Washington for Jobs and freedom through primary sources. Black History Bulletin, 75(2), 7-15. 
Schwarzmeier, J. (2001). Die Autonomen zwischen Subkultur und sozialer Bewegung. BoD-Books on Demand.

Staab, J. F. (1990). Nachrichtenwert-theorie; Formale struktur und empirische gehalt. Freiburg: Alber.

Suber, P. (1999). Civil disobedience. Philosophy of law: An encyclopedia. https://dash.harvard.edu/bitstream/handle/1/4725008/suber_civ-dis.htm?sequence =3, Zugangsdatum: 03.02.2021.

The Martin Luther King Jr. Institute. (2021). Beyond Vietnam. https://kinginstitute.stanford.edu/king-papers/documents/beyond-vietnam, Zugangsdatum : 01.02.2021.

Weber, J. R. (1996). Der US-amerikanische Kulturimperialismus und die schwarze Bürgerrechtsbewegungen; eine demokratische, politologische analyse. Universität Wien: Dissertation.

Wilk, M. (2020). Fragile kollektive Identitäten: Wie sich soziale Bewegungen radikalisieren (Vol. 238). transcript Verlag. 